\title{
Circular brass foil failed by plugging opposite to direction of incident laser beam
}

\author{
Y.C. Zhou ${ }^{\mathrm{a}, \mathrm{b}, *}$, Z.P. Duan ${ }^{\mathrm{a}}$ \\ a Laboratory For Material Dynamics \& Laser-Material Interaction, Institute of Mechanics, Chinese Academy of Science, Beijing, \\ 100080, China \\ ${ }^{\mathrm{b}}$ Department of Physics, Xiangtan University, 411105, Xiangtan Hunan, China
}

\begin{abstract}
The peripheries of circular foils of $30 \mathrm{~mm}$ in diameter and $0.1 \mathrm{~mm}$ thick are fixed while their surfaces are subjected to a long pulsed laser over a central region that may vary from $2 \mathrm{~mm}$ to $6 \mathrm{~mm}$ in diameter. Failure is observed and classified into three stages; they are referred to as thermal bulging, localized shear deformation, and perforation by plugging. A distinct feature of the failure mode is that bulging and plugging occurred in the direction opposite to the incident laser beam. Such a phenomenon can be expected to occur for a laser intensity threshold value of about $0.61 \times 10^{6} \mathrm{~W} / \mathrm{cm}^{2}$ beyond which local melting of the material begins to take place.
\end{abstract}

\section{Introduction}

Depending on the intensity and concentration of a laser beam over a solid, material damage could occur by spallation, melting and/or vaporization [1-5]. The precise mechanism of failure depends on the rate and intensity at which energy is transferred to the solid over a given region of a pre-assigned surface area in addition to the geometric configuration of the body. When laser energy is transmitted uniformly and wave reflection takes place between two surfaces, there is the potential of failure by spallation. Melting and/or vaporization are attributed to intensified energy applied over a short time period leaving

\footnotetext{
* Corresponding author. Tel. +861254 5533 (ext. 2201), fax +8612561284 .
}

no time for dissipation due to the creation of free surfaces or shear deformation.

Those situations prevail where the size and intensity of the laser beam are such that the spatial structure effect will contribute to the mode of failure such as bulging followed by plugging. Such are the cases considered in this investigation.

\section{Problem statement}

Consider an incident laser beam that impinges normally on a circular brass foil as shown in Fig. 1 . The $z$ - and $r$-direction are aligned normal and parallel to the specimen whose edge is clamped. Let $2 a$ denote the diameter of the laser spot that can vary from $2 \mathrm{~mm}$ to $6 \mathrm{~mm}$. The foil has a diameter of $30 \mathrm{~mm}$ and thickness of $0.1 \mathrm{~mm}$. The 


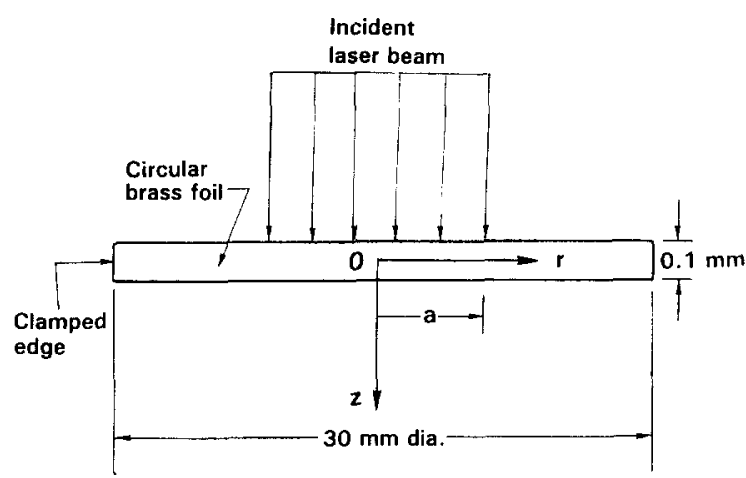

Fig. 1. Schematic of a normal incident laser beam impinging on a circular brass foil specimen.

brass material contains $65 \%$ copper, $33.6 \%$ zinc, $0.03 \%$ iron, $0.06 \%$ antimony and other microelements that are negligibly small in percentage by weight.

The energy of the laser beam ranges from $25 \mathrm{~J}$ to $40 \mathrm{~J}$ with an intensity of the order of $10^{5}$ to $10^{6}$ $\mathrm{W} / \mathrm{cm}^{2}$. It is a single pulse $\mathrm{Nd}$ : glass laser with a wavelength of $1.06 \mu \mathrm{m}$. Displayed in Figs. 2 and 3 are, respectively, the normalized intensity $I / I_{\max }$ with time and space coordinate $r / a$, where $r$ is the radial distance and $a$ the radius of the laser spot. The full width at half maximum of the laser is approximately $200 \mu \mathrm{s}$. The spatial distribution of the laser intensity is non-Gaussian but roughly uniform within the laser irradiated region and

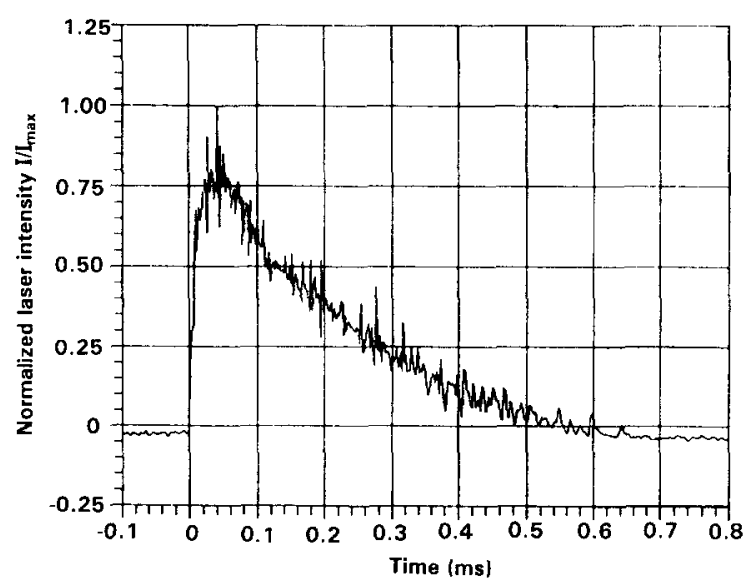

Fig. 2. Variations of the normalized laser intensity with time for a Nd: glass pulsed laser.

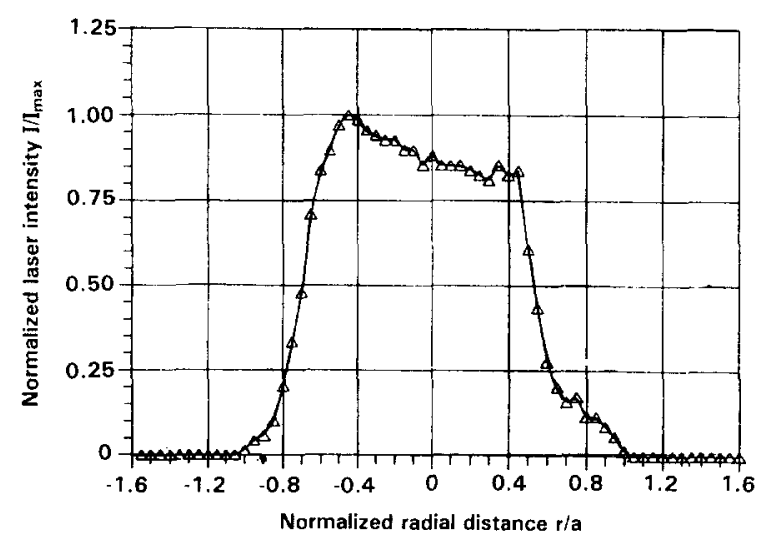

Fig. 3. Variations of the normalized laser intensity with dimensionless radial distance for a Nd:glass pulsed laser.

declines very sharply towards the edge where the laser spot terminates.

\section{Description of failure mode}

Illustrated schematically in Figs. 4 and 5 is the evolution of specimen failure. Fig. 4(a) shows bulging of the brass foil at the earlier stage of

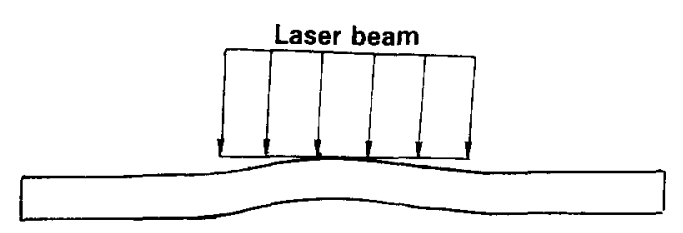

(a) Bulging

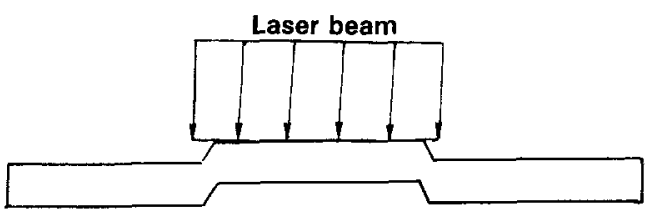

(b) Localized shear deformation

Fig. 4. Initial stages of damage evolution: (a) bulging and (b) localized shear deformation. 


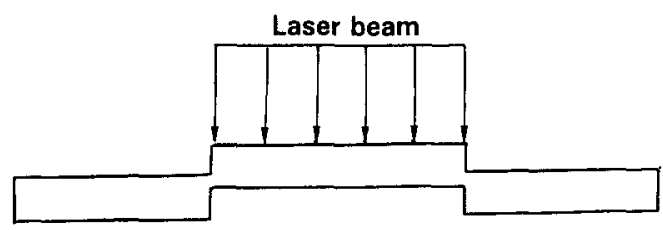

(a) Plugging intiation

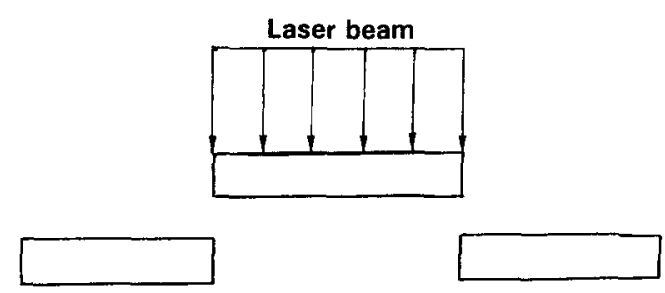

(b) Perforation

Fig. 5. Final stages of failure: (a) plugging initiation and (b) perforation.

laser irradiation. Note that this occurs towards the side of the incident laser beam where the temperature would be higher. Considerable shear deformation occurs around a rim near the outer edge of the laser beam. This leads to the softening of the material due to intense heating.

Further intensification of the energy around the periphery of the laser beam leads to the initiation of plugging and final perforation which are shown in Figs. 5(a) and 5(b), respectively. The plugging mode of failure is customarily known to be associated with metal projectiles penetrating through metal targets in plate form [3,4]. A plug of the target material is ejected in the direction of the energy source that is the moving projectile. In the case of an incident laser beam, plugging occurred opposite to the incoming direction of the energy source. The initial bulge occurs on the side with higher temperature that determines the direction of plugging.

\section{Observed damage evolution}

Scanning electron microscopy (SEM) showed that increased laser intensity led to the initiation of microcracks in the peripheral region with ra- dius $r \sim a$. The macrocracks appeared on the rear surface and then spread into the material. They would grow and coalesce into macrocracks that grow rapidly in the circumferential direction as well as in the thickness direction.

\subsection{Laser intensity of about $0.61 \times 10^{6} \mathrm{~W} / \mathrm{cm}^{2}$}

The photo of a polished section of brass specimen is shown in Fig. 6 bulging in the direction opposite to the laser beam is observed with a maximum center deflection of $0.03 \mathrm{~mm}$. This corresponded to a laser energy of $8.2 \mathrm{~J}$; an intensity greater than $0.61 \times 10^{6} \mathrm{~W} / \mathrm{cm}^{2}$; and a spot diameter of $2.3 \mathrm{~mm}$. Softening of the material prevailed around the outer edge of the laser spot. When the laser intensity is less than $0.51 \times 10^{6}$ $\mathrm{W} / \mathrm{cm}^{2}$, no visible macroscopic damage was observed on the front or rear surface of the brass specimen.

\subsection{Laser intensity of $0.75 \times 10^{6} \mathrm{~W} / \mathrm{cm}^{2}$}

When the laser intensity is increased to $0.75 \times$ $10^{6} \mathrm{~W} / \mathrm{cm}^{2}$ with an energy of $29 \mathrm{~J}$ and a laser spot diameter of $4.5 \mathrm{~mm}$, the brass foil is totally fractured and fragmented, a sectioned photo of which is shown in Fig. 7. A slight melting is observed on the front surface at the periphery of the laser spot.

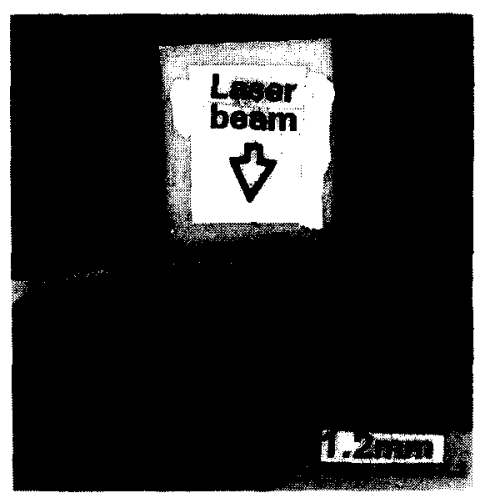

Fig. 6. Photo of sectioned brass foil at the bulge stage with a laser energy of $8.2 \mathrm{~J}$ over an area of $2.3 \mathrm{~mm}$ in diameter. 


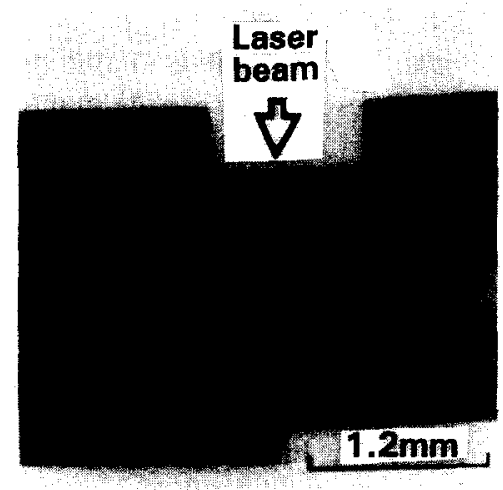

Fig. 7. Photo of fractured and fragmented brass foil subjected to a laser energy of $9.2 \mathrm{~J}$ over region $2.3 \mathrm{~mm}$ in diameter.

\subsection{Threshold intensity}

According to the test data in this study, the laser intensity threshold value $I_{\mathrm{cr}}$ for plugging to occur is about $0.61 \times 10^{6} \mathrm{~W} / \mathrm{cm}^{2}$. As $I$ exceeds $I_{\mathrm{cr}}$, local melting of the material begins to take place as in the case of $I=0.75 \times 10^{6} \mathrm{~W} / \mathrm{cm}^{2}$. A study on laser-induced spallation in $0.1 \mathrm{~mm}$ thick copper foil has been made in [5]. The intensity was of the order of $5.3 \times 10^{10} \mathrm{~W} / \mathrm{cm}^{2}$; it differed five orders of magnitude to failure by plugging which is about $0.71 \times 10^{6} \mathrm{~W} / \mathrm{cm}^{2}$. The thresholds for the laser energy do not distinguish the two different failure modes as they are both approximately equal to $210 \mathrm{~J} / \mathrm{cm}^{2}$.

\section{Conclusions}

The observed plugging failure of the brass foil specimen subjected to the $\mathrm{Nd}$ : glass long pulsed laser can be summarized in three stages.

Stage 1 - A high temperature state is built up on the specimen front surface impinged by a normal incident laser beam. This causes the foil to bulge towards the laser beam.

Stage 2 - A steep temperature gradient prevails across the periphery of the laser beam where the spot terminates. High shear deformation concentrated in a rim is developed and causes a circular region impinged upon by the laser to move further in the bulged direction.

Stage 3 - When the laser intensity reaches a critical value, failure by plugging takes place opposite to the direction of the incident laser beam.

On a microscopic scale, the plugging failure characteristics induced by a laser are similar to those found in brass plates perfected by metal projectiles. Such experiments are well known and have been carried out using a split Hopkinson pressure bar. To reiterate, laser-induced failure by plugging depends on the incident laser intensity and duration, the spatial profile of the laser beam and their relation to the geometric and material characteristics of the specimen, and see Ref. [6] for a detailed analysis.

\section{Acknowledgements}

Authors would like to express their sincere thanks to Profs. Z.M. Chen and B.M. Xie for their many helpful discussions during the preparation of this paper. Support for this research program was provided partly by NNSF of China and partly by the Field of Laser Technology, 863-NHT Research Development Program. Their support is also gratefully acknowledged.

\section{References}

[1] B.C.H. Wendlandt, Theoretical Studies of the Interation of High-Power Laser Beam and Metals, NASA-74-16188 (1973).

[2] A. Kar and J. Mazumder, Two-dimensional model for material damage due to melting and vaporization during laser irradiation, J. Appl. Phys. 68, 3884-3891 (1990).

[3] C.E. Anderson and S.R. Bodner, Ballistic impact: the status of analytical and numerical modeling, Int. J. Impact Engng. 7, 9-35 (1988).

[4] M. Shoukry, S. Nair and S. Kalpakjian, Effect of shear deformation on the dynamic plastic bending of metallic plates during normal penetration, Int. J. Engng. Sci. 29, 1035-1052 (1991).

[5] S. Eliezer, I. Gilath, T. Bar-Noy, Laser induced spall in metals: experiment and simulation, J. Appl. Phys. 67, 715724 (1990).

[6] Z.P. Duan, Y.C. Zhou and C.G. Huang, On laser-induced reverse plugging effect, Acta Mech. Sinica, 11 (1995) 239. 